\title{
Data Envelopment Analysis with Uncertain Inputs and Outputs
}

\author{
Meilin Wen, ${ }^{1,2}$ Linhan Guo, ${ }^{1,2}$ Rui Kang, ${ }^{1,2}$ and Yi Yang ${ }^{1,2}$ \\ ${ }^{1}$ Science and Technology on Reliability and Environmental Engineering Laboratory, Beijing 100191, China \\ ${ }^{2}$ School of Reliability and Systems Engineering, Beihang University, Beijing 100191, China \\ Correspondence should be addressed to Linhan Guo; linhanguo@buaa.edu.cn
}

Received 27 June 2014; Accepted 15 July 2014; Published 5 August 2014

Academic Editor: Xiang Li

Copyright (C) 2014 Meilin Wen et al. This is an open access article distributed under the Creative Commons Attribution License, which permits unrestricted use, distribution, and reproduction in any medium, provided the original work is properly cited.

\begin{abstract}
Data envelopment analysis (DEA), as a useful management and decision tool, has been widely used since it was first invented by Charnes et al. in 1978. On the one hand, the DEA models need accurate inputs and outputs data. On the other hand, in many situations, inputs and outputs are volatile and complex so that they are difficult to measure in an accurate way. The conflict leads to the researches of uncertain DEA models. This paper will consider DEA in uncertain environment, thus producing a new model based on uncertain measure. Due to the complexity of the new uncertain DEA model, an equivalent deterministic model is presented. Finally, a numerical example is presented to illustrate the effectiveness of the uncertain DEA model.
\end{abstract}

\section{Introduction}

Data envelopment analysis is a mathematical programming technique that measures the relative efficiency of decision making units with multiple inputs and outputs, which was initialized by Charnes et al. [1]. This was followed by variety of theory research work, including Banker et al. [2], Charnes et al. [3], Petersen [4], and Tone [5]. More DEA papers can refer to Seiford [6] in which 500 references are documented.

The original DEA models assume that inputs and outputs are measured by exact values. However, in many situations, such as in a manufacturing system, a production process, or a service system, inputs and outputs are volatile and complex so that they are difficult to measure in an accurate way. Thus many researchers tried to model DEA with various uncertain theories. Probability theory is the earliest theory which was used to establish the stochastic DEA models. Sengupta [7] generalized the stochastic DEA model using the expected value. Banker [8] incorporated statistical elements into DEA, thus developing a statistical method. Many papers [9-13] have employed the chance-constrained programming to DEA in order to accommodate stochastic variations in data. Fuzzy theory is another theory which was used to deal with the uncertainty in DEA. As one of the DEA initiators, Cooper et al. [14-16] introduced how to deal with imprecise data such as bounded data, ordinal data, and ratio bounded data in DEA. Kao and Liu [17] developed a method to find the membership functions of the fuzzy efficiency scores when some inputs or inputs are fuzzy numbers. Entani et al. [18] proposed a DEA model with an interval efficiency by the pessimistic and the optimistic values. Many researchers have introduced possibility measure [19] into DEA [20, 21].

A lot of surveys showed that human uncertainty does not behave like fuzziness. For example, we say "the input is about 10." Generally, we employ fuzzy variable to describe the concept of "about 10;" then there exists a membership function, such as a triangular one $(9,10,11)$. Based on this membership function, we can obtain that "the input is exactly 10 " with possibility measure 1 . On the other hand, the opposite event of "not exactly 10 " has the same possibility measure. The conclusion that "not 10" and "exactly 10 " have the same possibility measure is not appropriate. This inspired Liu [22] to found an uncertainty theory which has become a branch of axiomatic mathematics for modeling human uncertainty. This paper will apply the uncertainty theory to DEA to deal with human uncertainty, thus producing some uncertain DEA models.

In this paper, we will assume the inputs and outputs are uncertain variables and propose some uncertain DEA models. The rest of this paper is organized as follows. Section 2 
will introduce some basic concepts and properties about uncertain variables. Then an uncertain DEA model as well as its equivalent crisp model will be presented in Section 3. Finally, a numerical example will be given to illustrate the uncertain DEA model in Section 4.

\section{Preliminaries}

Uncertainty theory was founded by Liu [22] in 2007 and refined by Liu [23] in 2010. As extensions of uncertainty theory, uncertain process, and uncertain differential equations [24], uncertain calculus [25] were proposed. Besides, uncertain programming was first proposed by Liu [26] in 2009, which wants to deal with the optimal problems involving uncertain variable. This work was followed by an uncertain multiobjective programming, an uncertain goal programming [27], and an uncertain multilevel programming [28]. Since that, uncertainty theory was used to solve variety of real optimal problems, including finance [29-31], reliability analysis [32, 33], graph [34, 35], and train scheduling [36, 37]. In this section, we will state some basic concepts and results on uncertain variables. These results are crucial for the remainder of this paper.

Let $\Gamma$ be a nonempty set, and let $€$ be a $\sigma$-algebra over $\Gamma$. Each element $\Lambda \in \mathrm{E}$ is assigned a number $M\{\Lambda\} \in$ $[0,1]$. In order to ensure that the number $M\{\Lambda\}$ has certain mathematical properties, Liu [22] presented the four axioms.

Axiom 1. $M\{\Gamma\}=1$ for the universal set $\Gamma$.

Axiom 2. $M\{\Lambda\}+M\left\{\Lambda^{c}\right\}=1$ for any event $\Lambda$.

Axiom 3. For every countable sequence of events $\Lambda_{1}, \Lambda_{2}, \ldots$, we have

$$
M\left\{\bigcup_{i=1}^{\infty} \Lambda_{i}\right\} \leq \sum_{i=1}^{\infty} M\left\{\Lambda_{i}\right\}
$$

Axiom 4. Let $\left(\Gamma_{k}, \mathrm{E}_{k}, M_{k}\right)$ be uncertainty spaces for $k=$ $1,2, \ldots$. Then the product uncertain measure $M$ is an uncertain measure satisfying

$$
M\left\{\prod_{k=1}^{\infty} \Lambda_{k}\right\}=\bigwedge_{k=1}^{\infty} M_{k}\left\{\Lambda_{k}\right\}
$$

where $\Lambda_{k}$ are arbitrarily chosen events from $\iota_{k}$ for $k=$ $1,2, \ldots$, respectively.

If the set function $M$ satisfies the first three axioms, it is called an uncertain measure.

Definition 1 (see Liu [22]). Let $\Gamma$ be a nonempty set, let $€$ be a $\sigma$-algebra over $\Gamma$, and let $M$ be an uncertain measure. Then the triplet $(\Gamma, €, M)$ is called an uncertainty space.

Definition 2 (see Liu [22]). An uncertain variable $\xi$ is a measurable function from an uncertainty space $(\Gamma, €, M)$ to the set of real numbers; that is, for any Borel set $B$ of real numbers, the set

$$
\{\xi \in B\}=\{\gamma \in \Gamma \mid \xi(\gamma) \in B\}
$$

is an event.

Definition 3 (see Liu [22]). The uncertainty distribution $\Phi$ of an uncertain variable $\xi$ is defined by

$$
\Phi(x)=M\{\xi \leq x\}
$$

for any real number $x$.

Example 4. The linear uncertain variable $\xi \sim \mathscr{L}(a, b)$ has an uncertainty distribution

$$
\Phi(x)= \begin{cases}0, & \text { if } x \leq a, \\ \frac{(x-a)}{(b-a)}, & \text { if } a \leq x \leq b \\ 1, & \text { if } x \geq b .\end{cases}
$$

Example 5. An uncertain variable $\xi$ is called zigzag if it has a zigzag uncertainty distribution

$$
\Phi(x)= \begin{cases}0, & \text { if } x \leq a, \\ \frac{(x-a)}{2(b-a)}, & \text { if } a \leq x \leq b \\ \frac{(x+c-2 b)}{2(c-b)}, & \text { if } b \leq x \leq c, \\ 1, & \text { if } x \geq c\end{cases}
$$

denoted by $\mathscr{Z}(a, b, c)$, where $a, b, c$ are real numbers with $a<$ $b<c$.

Definition 6 (see Liu [25]). The uncertain variables $\xi_{1}, \xi_{2}, \ldots, \xi_{n}$ are said to be independent if

$$
M\left\{\bigcap_{i=1}^{n}\left(\xi_{i} \in B_{i}\right)\right\}=\bigwedge_{i=1}^{n} M\left\{\xi_{i} \in B_{i}\right\}
$$

for any Borel sets $B_{1}, B_{2}, \ldots, B_{n}$.

Definition 7 (see Liu [23]). An uncertainty distribution $\Phi$ of an uncertain variable $\xi$ is said to be regular if its inverse function $\Phi^{-1}(\alpha)$ exists and is unique for each $\alpha \in(0,1)$. In this case, the inverse function $\Phi^{-1}(\alpha)$ is called the inverse uncertainty distribution of $\xi$.

Example 8. The inverse uncertainty distribution of a zigzag uncertain variable $\mathscr{Z}(a, b, c)$ is

$$
\Phi^{-1}(\alpha)= \begin{cases}(1-2 \alpha) a+2 \alpha b, & \text { if } \alpha \leq 0.5 \\ (2-2 \alpha) b+(2 \alpha-1) c, & \text { if } \alpha>0.5\end{cases}
$$

Theorem 9 (see Liu [23]). Let $\xi_{1}, \xi_{2}, \ldots, \xi_{n}$ be independent uncertain variables with regular uncertainty distributions 
$\Phi_{1}, \Phi_{2}, \ldots, \Phi_{n}$, respectively. If $f$ is a strictly increasing function, then

$$
\xi=f\left(\xi_{1}, \xi_{2}, \ldots, \xi_{n}\right)
$$

is an uncertain variable with inverse uncertainty distribution

$$
\Psi^{-1}(\alpha)=f\left(\Phi_{1}^{-1}(\alpha), \Phi_{2}^{-1}(\alpha), \ldots, \Phi_{n}^{-1}(\alpha)\right) .
$$

Example 10. Let $\xi$ be an uncertain variable with regular uncertainty distribution $\Phi$. Since $f(x)=a x+b$ is a strictly increasing function for any constants $a>0$ and $b$, the inverse uncertainty distribution of $a \xi+b$ is

$$
\Psi^{-1}(\alpha)=a \Phi_{1}^{-1}(\alpha)+b .
$$

Example 11. Let $\xi_{1}, \xi_{2}, \ldots, \xi_{n}$ be independent uncertain variables with regular uncertainty distributions $\Phi_{1}, \Phi_{2}, \ldots, \Phi_{n}$, respectively. Since

$$
f\left(x_{1}, x_{2}, \ldots, x_{n}\right)=x_{1}+x_{2}+\cdots+x_{n}
$$

is a strictly increasing function, the sum

$$
\xi=\xi_{1}+\xi_{2}+\cdots+\xi_{n}
$$

is an uncertain variable with inverse uncertainty distribution

$$
\Psi^{-1}(\alpha)=\Phi_{1}^{-1}(\alpha)+\Phi_{2}^{-1}(\alpha)+\cdots+\Phi_{n}^{-1}(\alpha) .
$$

Theorem 12 (see Liu [23]). Assume the constraint function $g\left(x, \xi_{1}, \xi_{2}, \ldots, \xi_{n}\right)$ is strictly increasing with respect to $\xi_{1}, \xi_{2}, \ldots, \xi_{k}$ and strictly decreasing with respect to $\xi_{k+1}, \xi_{k+2}, \ldots, \xi_{n}$. If $\xi_{1}, \xi_{2}, \ldots, \xi_{n}$ are independent uncertain variables with uncertainty distributions $\Phi_{1}, \Phi_{2}, \ldots, \Phi_{n}$, respectively, then the chance constraint

$$
M\left\{g\left(x, \xi_{1}, \xi_{2}, \ldots, \xi_{n}\right) \leq 0\right\} \geq \alpha
$$

holds if and only if

$$
\begin{aligned}
& g\left(x, \Phi_{1}^{-1}(\alpha), \ldots, \Phi_{k}^{-1}(\alpha), \Phi_{k+1}^{-1}(1-\alpha), \ldots, \Phi_{n}^{-1}(1-\alpha)\right) \\
& \quad \leq 0 .
\end{aligned}
$$

\section{DEA Model}

In many situations, inputs and outputs are volatile and complex so that they are difficult to measure in an accurate way. This inspired many researchers to apply probability to DEA. As we know, probability or statistics needs a large amount of historical data. In the vast majority of real cases, the sample size is too small (even no sample) to estimate a probability distribution. Then we have to invite some domain experts to evaluate their degree of belief that each event will occur. This section will give some researches to empirical uncertain DEA using the theory introduced in Section 2. The new symbols and notations are given as follows:

$$
\mathrm{DMU}_{i} \text { : the } i \text { th DMU, } i=1,2, \ldots, n \text {; }
$$

$\mathrm{DMU}_{0}$ : the target DMU;

$\tilde{x}_{k}=\left(\widetilde{x}_{k 1}, \tilde{x}_{k 2}, \ldots, \widetilde{x}_{k p}\right)$ : the uncertain inputs vector of $\mathrm{DMU}_{k}, k=1,2, \ldots, n$;

$\Phi_{k i}(x)$ : the uncertainty distribution of $\tilde{x}_{k i}, k=$ $1,2, \ldots, n, i=1,2, \ldots, p$;

$\widetilde{x}_{0}=\left(\widetilde{x}_{01}, \widetilde{x}_{02}, \ldots, \widetilde{x}_{0 p}\right):$ the inputs vector of the target $\mathrm{DMU}_{0}$;

$\Phi_{0 i}(x)$ : the uncertainty distribution of $\tilde{x}_{0 i}, i=$ $1,2, \ldots, p$;

$\tilde{y}_{k}=\left(\widetilde{y}_{k 1}, \tilde{y}_{k 2}, \ldots, \tilde{y}_{k q}\right):$ the uncertain outputs vector of $\mathrm{DMU}_{k}, k=1,2, \ldots, n$;

$\Psi_{k j}(x)$ : the uncertainty distribution of $\tilde{y}_{k j}, k=$ $1,2, \ldots, n, j=1,2, \ldots, q$;

$\tilde{y}_{0}=\left(\tilde{y}_{01}, \tilde{y}_{02}, \ldots, \tilde{y}_{0 q}\right)$ : the outputs vector of the target $\mathrm{DMU}_{0}$;

$\Psi_{0 j}(x)$ : the uncertainty distribution of $\tilde{y}_{0 j}, j=$ $1,2, \ldots, q$.

3.1. Uncertainty Distributions of Inputs and Outputs. Liu and $\mathrm{Ha}$ [38] proposed a questionnaire survey for collecting expert's experimental data. It is based on expert's experimental data rather than historical data. The starting point is to invite one expert who is asked to complete a questionnaire about the meaning of an uncertain input (output) $\xi$ like "How many is the input (output)."

We first ask the domain expert to choose a possible value $x$ that the uncertain input $\xi$ may take and then quiz him,

"How likely is $\xi$ less than or equal to $x$ ?"

Denote the expert's belief degree by $\alpha$. An expert's experimental data $(x, \alpha)$ is thus acquired from the domain expert.

Repeating the above process, we can obtain the following expert's experimental data:

$$
\left(x_{1}, \alpha_{1}\right),\left(x_{2}, \alpha_{2}\right), \ldots,\left(x_{n}, \alpha_{n}\right)
$$

that meet the following consistence condition (perhaps after a rearrangement):

$$
x_{1}<x_{2}<\cdots<x_{n}, \quad 0 \leq \alpha_{1} \leq \alpha_{2} \leq \cdots \alpha_{n} \leq 1 .
$$

Based on those expert's experimental data, Liu and Ha [38] suggested an empirical uncertainty distribution,

$\Phi(x)$

$$
= \begin{cases}0, & \text { if } x \leq x_{1}, \\ \alpha_{i}+\frac{\left(\alpha_{i+1}-\alpha_{i}\right)\left(x-x_{i}\right)}{x_{i+1}-x_{i}}, & \text { if } x_{i} \leq x \leq x_{i+1}, 1 \leq i<n, \\ 1, & \text { if } x>x_{n} .\end{cases}
$$

Assume there are $m$ domain experts and each produces an uncertainty distribution. Then we may get $m$ uncertainty distributions $\Phi_{1}(x), \Phi_{2}(x), \ldots, \Phi_{m}(x)$. The Delphi method 
was originally developed in the 1950s by the RAND Corporation based on the assumption that group experience is more valid than individual experience. Wang et al. [39] recast the Delphi method as a process to determine the uncertainty distribution. The main steps are listed as follows.

Step 1. The $m$ domain experts provide their expert's experimental data,

$$
\left(x_{i j}, \alpha_{i j}\right), \quad j=1,2, \ldots, n_{i}, i=1,2, \ldots, m .
$$

Step 2. Use the $i$ th expert's experimental data $\left(x_{i 1}, \alpha_{i 1}\right),\left(x_{i 2}, \alpha_{i 2}\right), \ldots,\left(x_{i n_{i}}, \alpha_{i n_{i}}\right)$ to generate the $i$ th expert's uncertainty distribution $\Phi_{i}$.

Step 3. Compute $\Phi(x)=w_{1} \Phi_{1}(x)+w_{2} \Phi_{2}(x)+\cdots+w_{m} \Phi_{m}(x)$, where $w_{1}, w_{2}, \ldots, w_{m}$ are convex combination coefficients.

Step 4. If $\left|\alpha_{i j}-\Phi\left(x_{i j}\right)\right|$ are less than a given level $\varepsilon>0$, then go to Step 5. Otherwise, the $i$ th expert receives the summary ( $\Phi$ and reasons) and then provides a set of revised expert's experimental data. Go to Step 2.

Step 5. The last $\Phi$ is the uncertainty distribution of the input (output).

3.2. Uncertain DEA Model. Similar to traditional DEA model [3], the objective of the uncertain DEA model is to maximize the total slacks in inputs and outputs subject to the constraints. Then the uncertain DEA model can be given as follows:

$$
\max \quad \sum_{i=1}^{p} s_{i}^{-}+\sum_{j=1}^{q} s_{j}^{+}
$$

subject to: $M\left\{\sum_{k=1}^{n} \tilde{x}_{k i} \lambda_{k} \leq \tilde{x}_{0 i}-s_{i}^{-}\right\} \geq \alpha, \quad i=1,2, \ldots, p$,

$$
M\left\{\sum_{k=1}^{n} \tilde{y}_{k j} \lambda_{k} \geq \tilde{y}_{0 j}+s_{j}^{+}\right\} \geq \alpha, \quad j=1,2 \ldots, q,
$$$$
\sum_{k=1}^{n} \lambda_{k}=1
$$

$$
\begin{aligned}
& \lambda_{k} \geq 0, \quad k=1,2, \ldots, n, \\
& s_{i}^{-} \geq 0, \quad i=1,2 \ldots, p, \\
& s_{j}^{+} \geq 0, \quad j=1,2, \ldots, q .
\end{aligned}
$$

Definition 13 ( $\alpha$-efficiency). $\mathrm{DMU}_{0}$ is $\alpha$-efficient if $s_{i}^{-*}$ and $s_{j}^{+*}$ are zero for $i=1,2, \ldots, p$ and $j=1, \ldots, q$, where $s_{i}^{-*}$ and $s_{j}^{+*}$ are optimal solutions of (21).

Since the uncertain measure is involved, this definition is different from traditional efficiency definition. For instance, as determined by the choice of $\alpha$, there is a risk that $\mathrm{DMU}_{0}$ will not be efficient even when the condition of Definition 13 is satisfied.

Since $j=0$ is one of the $\mathrm{DMU}_{j}$, we can always get a solution with $\lambda_{0}=1, \lambda_{j}=0(j \neq 0)$, and all slacks zero. Thus this uncertain DEA model has feasible solution and the optimal value $s_{i}^{-*}=s_{j}^{+*}=0$ for all $i, j$.

3.3. Deterministic Model. Model (21) is an uncertain programming model, which is too complex to compute directly. This section will give its equivalent crisp model to simplify the computation process.

Theorem 14. Assume that $\tilde{x}_{1 i}, \tilde{x}_{2 i}, \ldots, \tilde{x}_{n i}$ are independent uncertain inputs with uncertainty distribution $\Phi_{1 i}, \Phi_{2 i}, \ldots, \Phi_{n i}$ for each $i, i=1,2, \ldots, p$, and $\tilde{y}_{1 i}, \tilde{y}_{2 i}, \ldots, \tilde{y}_{n i}$ are independent uncertain outputs with uncertainty distribution $\Psi_{1 j}, \Psi_{2 j}, \ldots, \Psi_{n j}$ for each $j, j=1,2, \ldots, q$. Then

$$
\begin{aligned}
& M\left\{\sum_{k=1}^{n} \tilde{x}_{k i} \lambda_{k} \leq \tilde{x}_{0 i}-s_{i}^{-}\right\} \geq \alpha, \quad i=1,2, \ldots, p, \\
& M\left\{\sum_{k=1}^{n} \tilde{y}_{k j} \lambda_{k} \geq \tilde{y}_{0 j}+s_{j}^{+}\right\} \geq \alpha, \quad j=1,2 \ldots, q
\end{aligned}
$$

holds if and only if

$$
\begin{array}{r}
\sum_{k=1, k \neq 0}^{n} \lambda_{k} \Phi_{k i}^{-1}(\alpha)+\lambda_{0} \Phi_{0 i}^{-1}(1-\alpha) \leq \Phi_{0 i}^{-1}(1-\alpha)-s_{i}^{-}, \\
i=1,2, \ldots, p \\
\sum_{k=1, k \neq 0}^{n} \lambda_{k} \Psi_{k j}^{-1}(1-\alpha)+\lambda_{0} \Psi_{0 j}^{-1}(\alpha) \geq \Psi_{0 j}^{-1}(\alpha)+s_{j}^{-}, \\
j=1,2, \ldots, q .
\end{array}
$$

Proof. Without loss of generality, let $i=1$ and $x_{0}=x_{1}$; then we will consider the equation

$$
M\left\{\sum_{k=1}^{n} \tilde{x}_{k 1} \lambda_{k} \leq \tilde{x}_{11}-s_{i}^{-}\right\} \geq \alpha
$$

Rewrite (24) as

$$
M\left\{\sum_{k=2}^{n} \tilde{x}_{k 1} \lambda_{k}-\left(1-\lambda_{1}\right) \tilde{x}_{11} \leq-s_{i}^{-}\right\} \geq \alpha .
$$

Since $-\left(1-\lambda_{1}\right) \tilde{x}_{11}$ is an uncertain variable which is decreasing with respect to $\tilde{x}_{11}$, its inverse uncertainty distribution is

$$
\Upsilon_{11}^{-1}(\alpha)=-\left(1-\lambda_{1}\right) \Phi_{11}^{-1}(1-\alpha), \quad 0<\alpha<1 .
$$

For each $2 \leq k \leq n, \tilde{x}_{k 1} \lambda_{k}$ is an uncertain variable whose inverse uncertainty distribution is

$$
\Upsilon_{k 1}^{-1}(\alpha)=\lambda_{k} \Phi_{k 1}^{-1}(\alpha), \quad 0<\alpha<1
$$


TABLE 1: DMUs with two uncertain inputs and two uncertain outputs.

\begin{tabular}{|c|c|c|c|c|c|}
\hline $\mathrm{DMU}_{i}$ & 1 & 2 & 3 & 4 & 5 \\
\hline Input 1 & $\mathscr{Z}(3.5,4.0,4.5)$ & $\mathscr{Z}(2.9,2.9,2.9)$ & $\mathscr{Z}(4.4,4.9,5.4)$ & $\mathscr{Z}(3.4,4.1,4.8)$ & $\mathscr{Z}(5.9,6.5,7.1)$ \\
\hline Input 2 & $\mathscr{Z}(2.9,3.1,3.3)$ & $\mathscr{Z}(1.4,1.5,1.6)$ & $\mathscr{Z}(3.2,3.6,4.0)$ & $\mathscr{Z}(2.1,2.3,2.5)$ & $\mathscr{Z}(3.6,4.1,4.6)$ \\
\hline Output 1 & $\mathscr{Z}(2.4,2.6,2.8)$ & $\mathscr{Z}(2.2,2.2,2.2)$ & $\mathscr{Z}(2.7,3.2,3.7)$ & $\mathscr{Z}(2.5,2.9,3.3)$ & $\mathscr{Z}(4.4,5.1,5.8)$ \\
\hline Output 2 & $\mathscr{Z}(3.8,4.1,4.4)$ & $\mathscr{Z}(3.3,3.5,3.7)$ & $\mathscr{Z}(4.3,5.1,5.9)$ & $\mathscr{Z}(5.5,5.7,5.9)$ & $\mathscr{Z}(6.5,7.4,8.3)$ \\
\hline
\end{tabular}

TABLE 2: Results of evaluating the DMUs with $\alpha=0.6$.

\begin{tabular}{|c|c|c|c|}
\hline DMUs & $\left(\lambda_{1}^{*}, \lambda_{2}^{*}, \lambda_{3}^{*}, \lambda_{4}^{*}, \lambda_{5}^{*}\right)$ & $\sum_{i=1}^{p} s_{i}^{-*}+\sum_{j=1}^{q} s_{j}^{+*}$ & The result of evaluating \\
\hline $\mathrm{DMU}_{1}$ & $(0,0.25,0,0.75,0)$ & 1.89 & Inefficiency \\
\hline $\mathrm{DMU}_{2}$ & $(0,1,0,0,0)$ & 0 & Efficiency \\
\hline $\mathrm{DMU}_{3}$ & $(0,0,0,0.78,0.22)$ & 1.54 & Inefficiency \\
\hline $\mathrm{DMU}_{4}$ & $(0,0,0,1,0)$ & 0 & Efficiency \\
\hline $\mathrm{DMU}_{5}$ & $(0,0,0,0,1)$ & 0 & Efficiency \\
\hline
\end{tabular}

It follows from the operational law that the inverse uncertainty distribution of the sum $\sum_{k=2}^{n} \tilde{x}_{k 1} \lambda_{k}-\left(1-\lambda_{1}\right) \tilde{x}_{11}$ is

$$
\begin{aligned}
\Upsilon^{-1}(\alpha) & =\sum_{k=1}^{n} \Upsilon_{21}^{-1}(\alpha) \\
& =\sum_{k=2}^{n} \lambda_{k} \Phi_{k 1}^{-1}(\alpha)-\left(1-\lambda_{1}\right) \Phi_{11}^{-1}(1-\alpha), \quad 0<\alpha<1 .
\end{aligned}
$$

From which we may derive the result immediately for $i=1$ and $x_{0}=x_{1}$. Similarly, we can get other results.

Following Theorem 14, the uncertain DEA model can be converted to the crisp model as follows:

$$
\begin{array}{ll}
\max & \sum_{i=1}^{p} s_{i}^{-}+\sum_{j=1}^{q} s_{j}^{+} \\
\text {subject to: } \quad \sum_{k=1, k \neq 0}^{n} \lambda_{k} \Phi_{k i}^{-1}(\alpha)+\lambda_{0} \Phi_{0 i}^{-1}(1-\alpha) \\
\leq \Phi_{0 i}^{-1}(1-\alpha)-s_{i}^{-}, \quad i=1,2, \ldots, p, \\
\sum_{k=1, k \neq 0}^{n} \lambda_{k} \Psi_{k j}^{-1}(1-\alpha)+\lambda_{0} \Psi_{0 j}^{-1}(\alpha) \\
\geq \Psi_{0 j}^{-1}(\alpha)+s_{j}^{+}, \quad j=1,2, \ldots, q, \\
\sum_{k=1}^{n} \lambda_{k}=1, \\
\lambda_{k} \geq 0, \quad k=1,2, \ldots, n \\
s_{i}^{-} \geq 0, \quad i=1,2 \ldots, p, \\
s_{j}^{+} \geq 0, \quad j=1,2, \ldots, q
\end{array}
$$

which is a linear programming model. Thus it can be easily solved by many traditional methods.

\section{A Numerical Example}

This example wants to illustrate the uncertain DEA model. For simplicity, we will only consider five DMUs with two inputs and two outputs which are all zigzag uncertain variables denoted by $\mathscr{Z}(a, b, c)$. Table 1 gives the information of the DMUs.

For illustration, let $\mathrm{DMU}_{1}$ be the target DMU; then the uncertain DEA model (29) can be written as

$$
\begin{array}{ll}
\max & s_{1}^{-}+s_{2}^{-}+s_{1}^{+}+s_{2}^{+} \\
\text {subject to: } \quad & \sum_{k=2}^{5} \lambda_{k} \Phi_{k 1}^{-1}(\alpha)+\lambda_{1} \Phi_{11}^{-1}(1-\alpha) \\
& \leq \Phi_{11}^{-1}(1-\alpha)-s_{1}^{-}, \\
& \sum_{k=2}^{5} \lambda_{k} \Phi_{k 2}^{-1}(\alpha)+\lambda_{1} \Phi_{12}^{-1}(1-\alpha) \\
& \leq \Phi_{12}^{-1}(1-\alpha)-s_{2}^{-}, \\
& \sum_{k=2}^{5} \lambda_{k} \Psi_{k 1}^{-1}(1-\alpha)+\lambda_{1} \Psi_{11}^{-1}(\alpha) \geq \Psi_{11}^{-1}(\alpha)+s_{1}^{+}, \\
& \sum_{k=2}^{5} \lambda_{k} \Psi_{k 2}^{-1}(1-\alpha)+\lambda_{1} \Psi_{12}^{-1}(\alpha) \geq \Psi_{12}^{-1}(\alpha)+s_{2}^{+}, \\
& \sum_{k=1}^{5} \lambda_{k}=1, \\
& \lambda_{k} \geq 0, \quad k=1,2, \ldots, 5,
\end{array}
$$


TABLE 3: Results of evaluating the DMUs with different confidence level $\alpha$.

\begin{tabular}{llllll}
\hline$\alpha$ & DMU $_{1}$ & DMU $_{2}$ & DMU $_{3}$ & DMU $_{4}$ & DMU $_{5}$ \\
\hline 0.5 & Inefficiency & Efficiency & Inefficiency & Efficiency & Efficiency \\
0.6 & Inefficiency & Efficiency & Inefficiency & Efficiency \\
0.7 & Inefficiency & Efficiency & Inefficiency & Efficiency \\
0.8 & Inefficiency & Efficiency & Efficiency & Efficiency & Efficiency \\
0.9 & Efficiency & Efficiency & Efficiency & Efficiency & \\
\hline
\end{tabular}

Table 2 shows the results of evaluating DMUs with confidence level $\alpha=0.6$. The results can be interpreted in the following way: $\mathrm{DMU}_{1}$ and $\mathrm{DMU}_{3}$ are inefficient, whereas $\mathrm{DMU}_{2}, \mathrm{DMU}_{4}$, and $\mathrm{DMU}_{5}$ are efficient. Moreover, $\mathrm{DMU}_{3}$ is more efficient than $\mathrm{DMU}_{1}$ from the total slacks $\sum_{i=1}^{p} s_{i}^{-*}+$ $\sum_{j=1}^{q} s_{j}^{+*}$, since they are both inefficient.

Uncertain efficiencies obtained from model (30) for different confidence levels $\alpha$ are shown in Table $3 . \mathrm{DMU}_{1}$ is inefficient at all confidence levels, whereas $\mathrm{DMU}_{2}, \mathrm{DMU}_{4}$, and $\mathrm{DMU}_{5}$ are always efficient at all levels. It can be seen that the number of the efficient DMUs is affected by the confidence level $\alpha$. The higher the confidence level $\alpha$ is, the bigger the number of efficient DMUs is. This phenomena indicate that uncertain DEA is more complex than the traditional DEA because of the inherent uncertainty contained in inputs and outputs.

\section{Conclusion}

Due to its widely practical used background, data envelopment analysis (DEA) has become a pop area of research. Since the data cannot be precisely measured in some practical cases, many papers have been published when the inputs and outputs are uncertain. This paper has given some researches to uncertain DEA model. A new DEA model as well as its equivalent deterministic model was presented. For illustration, a numerical example was designed.

\section{Conflict of Interests}

The authors declare that they have no conflict of interests regarding the publication of this paper.

\section{Acknowledgment}

This work was supported by National Natural Science Foundation of China (nos. 71201005 and 61104132).

\section{References}

[1] A. Charnes, W. W. Cooper, and E. Rhodes, "Measuring the efficiency of decision making units," European Journal of Operational Research, vol. 2, no. 6, pp. 429-444, 1978.

[2] R. D. Banker, A. Charnes, and W. W. Cooper, "Some models for estimating technical and scale efficiencies in data envelopment analysis," Management Science, vol. 30, no. 9, pp. 1078-1092, 1984.

[3] A. Charnes, W. W. Cooper, B. Golany, L. Seiford, and J. Stutz, "Foundations of data envelopment analysis for ParetoKoopmans efficient empirical production functions," Journal of Econometrics, vol. 30, no. 1-2, pp. 91-107, 1985.

[4] N. C. Petersen, "Data envelopment analysis on a relaxed set of assumptions," Management Science, vol. 36, no. 3, pp. 305-314, 1990.

[5] K. Tone, "A slacks-based measure of efficiency in data envelopment analysis," European Journal of Operational Research, vol. 130, no. 3, pp. 498-509, 2001.

[6] L. M. Seiford, "A DEA bibliography (1978-1992)," in Data Envelopment Analysis: Theory, Methodology and Applications, A. Charnes, W. W. Cooper, A. Lewin, and L. Seiford, Eds., Kluwer Academic Publishers, Boston, Mass, USA, 1994.

[7] J. K. Sengupta, "Efficiency measurement in stochastic inputoutput systems," International Journal of Systems Science, vol. 13, no. 3, pp. 273-287, 1982.

[8] R. D. Banker, "Maximum likelihood, consistency and data envelopment analysis. A statistical foundation," Management Science, vol. 39, no. 10, pp. 1265-1273, 1993.

[9] O. Olesen and N. C. Petersen, "Chance constrained efficiency evaluation," Management Science, vol. 141, pp. 442-457, 1995.

[10] R. D. Banker, Stochastic Data Envelopment Analysis, CarnegieMellon University, Pittsburgh, Pennsylvania, 1986.

[11] S. Grosskopf, "Statistical inference and nonparametric efficiency: a selective survey," Journal of Productivity Analysis, vol. 7, no. 2-3, pp. 161-176, 1996.

[12] W. W. Cooper, Z. Huang, and S. X. Li, "Satisficing DEA models under chance constraints," Annals of Operations Research, vol. 66, pp. 279-295, 1996.

[13] W. W. Cooper, Z. M. Huang, V. Lelas, S. X. Li, and O. B. Olesen, "Chance constrained programming formulations for stochastic characterizations of efficiency and dominance in DEA," Journal of Productivity Analysis, vol. 9, no. 1, pp. 53-79, 1998.

[14] W. W. Cooper, K. S. Park, and G. Yu, "Idea and AR-IDEA: models for dealing with imprecise data in DEA," Management Science, vol. 45, no. 4, pp. 597-607, 1999.

[15] W. W. Cooper, K. S. Park, and G. Yu, "An illustrative application of idea (imprecise data envelopment analysis) to a Korean mobile telecommunication company," Operations Research, vol. 49 , no. 6, pp. 807-820, 2001. 
[16] W. W. Cooper, K. S. Park, and G. Yu, "IDEA (imprecise data envelopment analysis) with CMDs (column maximum decision making units)," The Journal of the Operational Research Society, vol. 52, no. 2, pp. 176-181, 2001.

[17] C. Kao and S. Liu, "Fuzzy efficiency measures in data envelopment analysis," Fuzzy Sets and Systems, vol. 113, no. 3, pp. 427437, 2000.

[18] T. Entani, Y. Maeda, and H. Tanaka, "Dual models of interval DEA and its extension to interval data," European Journal of Operational Research, vol. 136, no. 1, pp. 32-45, 2002.

[19] L. A. Zadeh, "Fuzzy sets as a basis for a theory of possibility," Fuzzy Sets and Systems, vol. 1, no. 1, pp. 3-28, 1978.

[20] P. Guo and H. Tanaka, "Fuzzy DEA: a perceptual evaluation method," Fuzzy Sets and Systems, vol. 119, no. 1, pp. 149-160, 2001.

[21] S. Lertworasirikul, S. Fang, J. A. Joines, and H. L. W. Nuttle, "Fuzzy data envelopment analysis (DEA): a possibility approach," Fuzzy Sets and Systems, vol. 139, no. 2, pp. 379-394, 2003.

[22] B. Liu, Uncertainty Theory, Springer, Berlin, Germany, 2nd edition, 2007

[23] B. Liu, Uncertainty Theory: A Branch of Mathematics for Modeling Human Uncertainty, Springer, Berlin, Germany, 2010.

[24] B. Liu, "Fuzzy process, hybrid process and uncertain process," Journal of Uncertain Systems, vol. 2, no. 1, pp. 3-16, 2008.

[25] B. Liu, "Some research problems in uncertainty theory," Journal of Uncertain Systems, vol. 3, no. 1, pp. 3-10, 2009.

[26] B. Liu, Theory and Practice of Uncertain Programming, Springer, Berlin, Germany, 2nd edition, 2009.

[27] B. Liu and X. W. Chen, "Uncertain multiobjective programming and uncertain goal programming," Tech. Rep., 2013.

[28] B. Liu and K. Yao, "Uncertain multilevel programming: algorithm and application," http://orsc.edu.cn/online/120114.pdf.

[29] X. Chen and B. Liu, "Existence and uniqueness theorem for uncertain differential equations," Fuzzy Optimization and Decision Making, vol. 9, no. 1, pp. 69-81, 2010.

[30] J. Peng and K. Yao, "A new option pricing model for stocks in uncertainty markets," International Journal of Operations Research, vol. 7, no. 4, pp. 213-224, 2010.

[31] B. Liu, "Extreme value theorems of uncertain process with application to insurance risk model," Soft Computing, vol. 17, no. 4, pp. 549-556, 2013.

[32] B. Liu, "Uncertain risk analysis and uncertain reliability analysis," Journal of Uncertain Systems, vol. 4, no. 3, pp. 163-170, 2010.

[33] Z. G. Zeng, M. L. Wen, and R. Kang, "Belief reliability: a new metrics for products'reliability," Fuzzy Optimization and Decision Making, vol. 12, no. 1, pp. 15-27, 2013.

[34] X. L. Gao, "Cycle index of uncertain graph," Information, vol. 16, no. 2, pp. 1131-1138, 2013.

[35] X. L. Gao and Y. Gao, "Connectedness index of uncertain graph," International Journal of Uncertainty, Fuzziness and Knowlege-Based Systems, vol. 21, no. 1, pp. 127-137, 2013.

[36] X. Li, D. Wang, K. Li, and Z. Gao, "A green train scheduling model and fuzzy multi-objective optimization algorithm," Applied Mathematical Modelling, vol. 37, no. 4, pp. 2063-2073, 2013.

[37] X. Li and K. Lo Hong, "An energy-efficient scheduling and speed control approach for metro rail operations," Transportation Research B: Methodological, vol. 64, pp. 73-89, 2014.
[38] Y.H. Liu and M. H. Ha, "Expected value of function of uncertain variables," Journal of Uncertain Systems, vol. 13, pp. 181-186, 2010.

[39] X. S. Wang, Z. C. Gao, and H. Y. Guo, "Delphi method for estimating uncertainty distributions," Information. An International Interdisciplinary Journal, vol. 15, no. 2, pp. 449-460, 2012. 


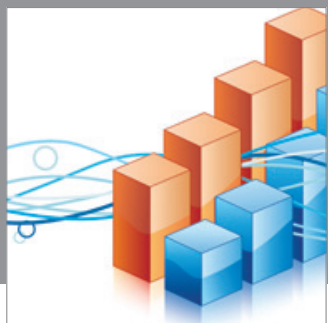

Advances in

Operations Research

mansans

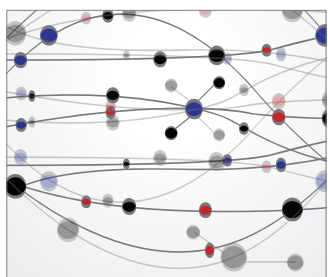

The Scientific World Journal
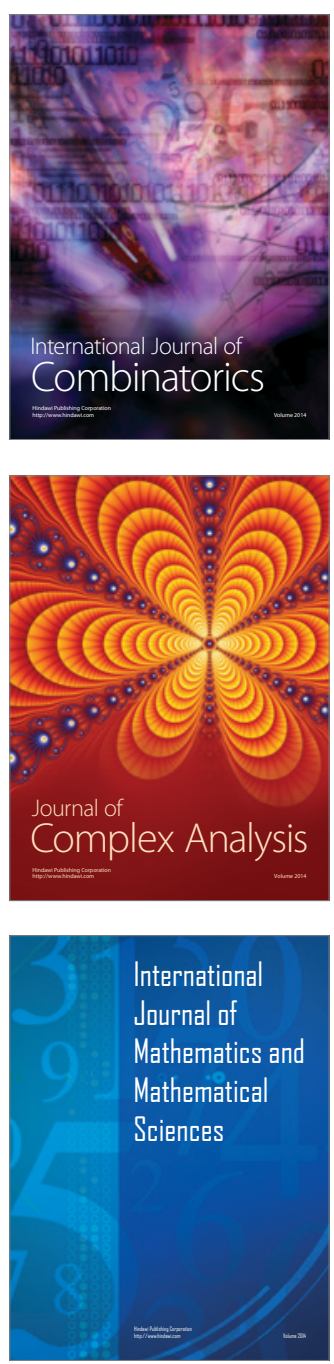
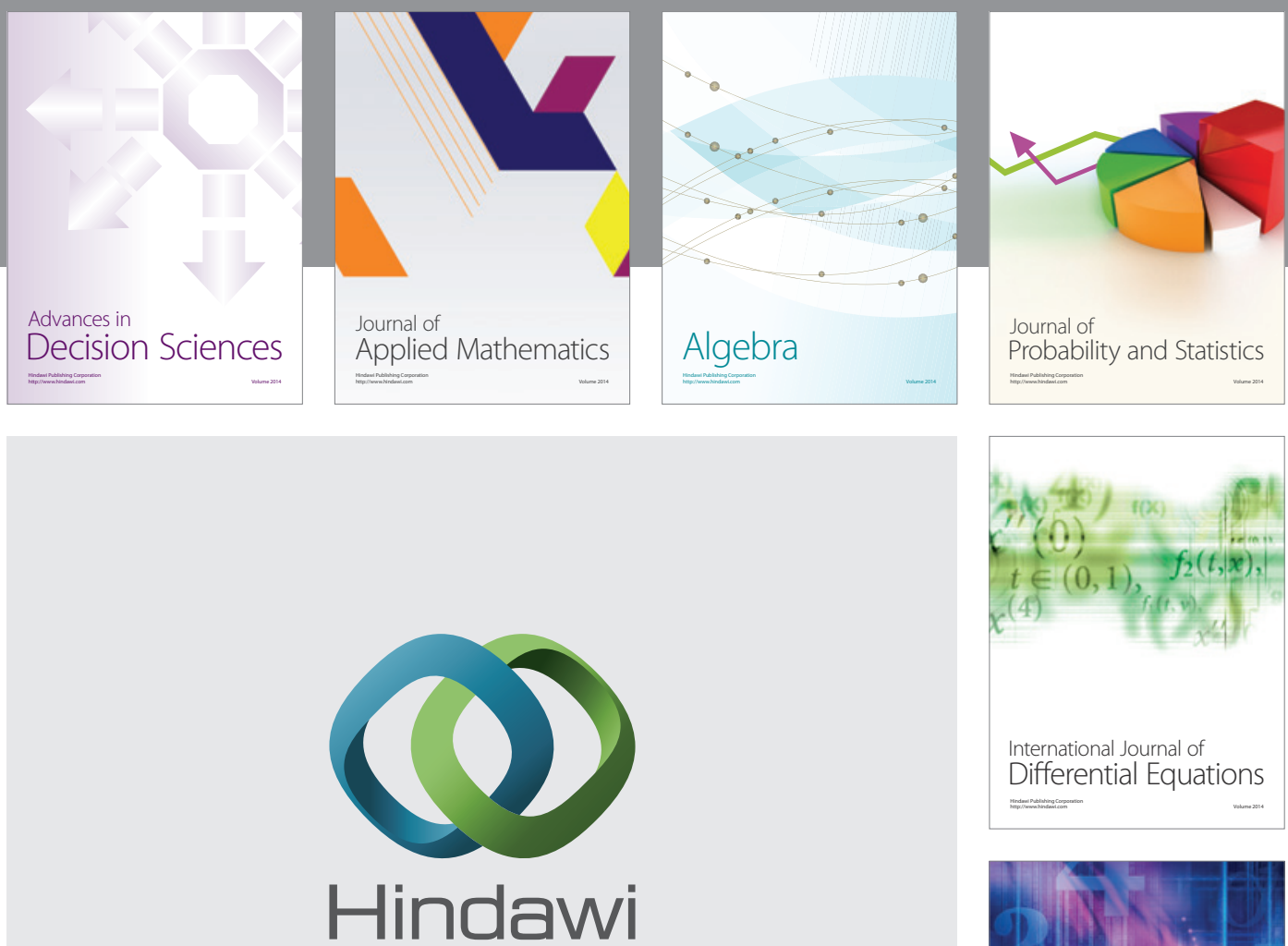

Submit your manuscripts at http://www.hindawi.com
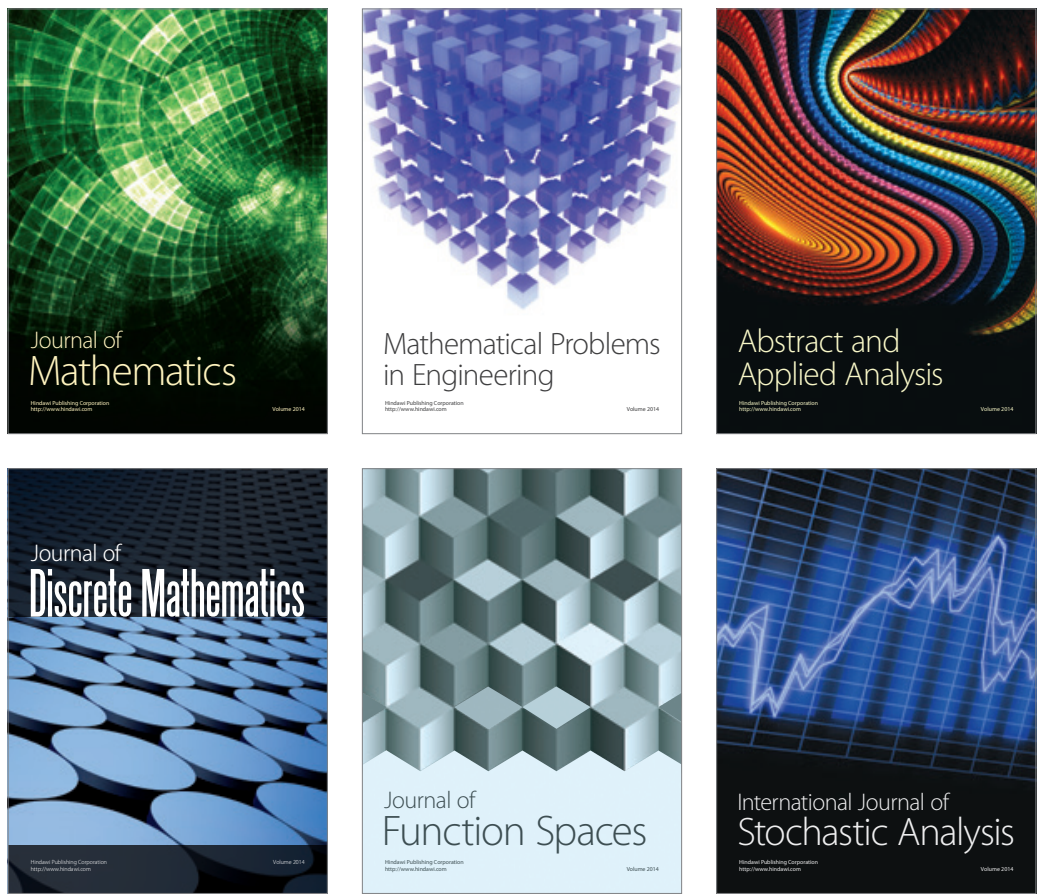

Journal of

Function Spaces

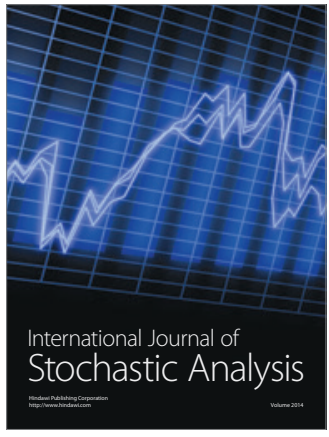

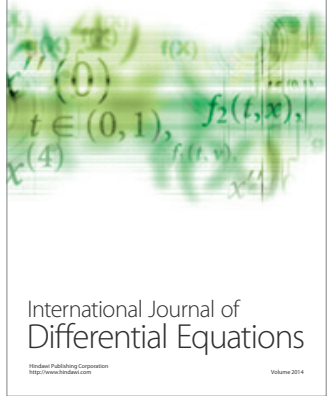
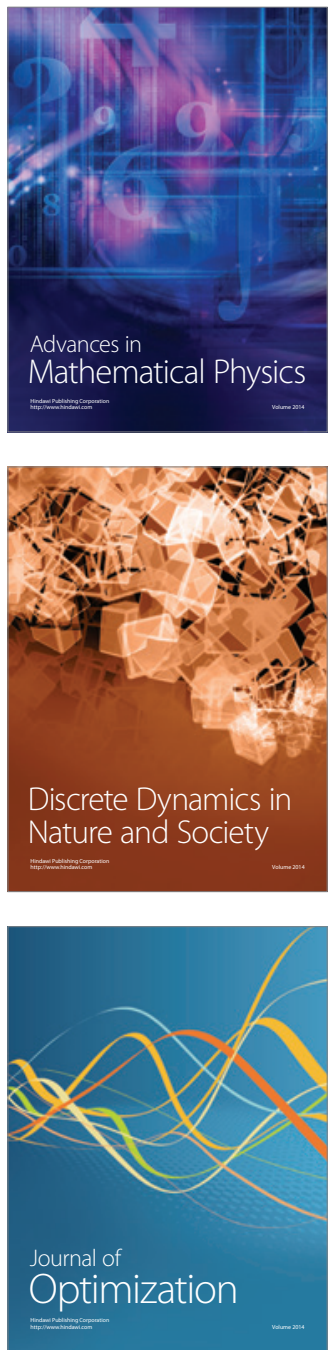